\title{
Measurement of two-phase flow and heat transfer parameters using infrared thermometry
}

\author{
Tae Hoon Kim, Eric Kommer, Serguei Dessiatoun, Jungho Kim* \\ University of Maryland, Department of Mechanical Engineering, College Park, MD 20742, USA
}

\section{A R T I C L E I N F O}

\section{Article history:}

Received 30 September 2011

Received in revised form 28 November 2011

Accepted 28 November 2011

Available online 9 December 2011

\section{Keywords:}

Infrared thermography

Heat transfer distribution

Two-phase flows

Droplet evaporation flow boiling

Liquid film thickness

\begin{abstract}
A B S T R A C T
A novel technique to measure heat transfer and liquid film thickness distributions over relatively large areas for two-phase flow and heat transfer phenomena using infrared (IR) thermometry is described. IR thermometry is an established technology that can be used to measure temperatures when optical access to the surface is available in the wavelengths of interest. In this work, a midwave IR camera (3.6-5.1 $\mu \mathrm{m})$ is used to determine the temperature distribution within a multilayer consisting of a silicon substrate coated with a thin insulator. Since silicon is largely transparent to IR radiation, the temperature of the inner and outer walls of the multilayer can be measured by coating selected areas with a thin, IR opaque film. If the fluid used is also partially transparent to IR, the flow can be visualized and the liquid film thickness can be measured. The theoretical basis for the technique is given along with a description of the test apparatus and data reduction procedure. The technique is demonstrated by determining the heat transfer coefficient distributions produced by droplet evaporation and flow boiling heat transfer.
\end{abstract}

(C) 2011 Elsevier Ltd. All rights reserved.

\section{Introduction}

Definitive understanding of phase change heat transfer mechanisms remains elusive due its sensitivity to many variables, but also due to a lack of reliable local information that can enable models to be tested. Although point measurements or area averaged measurements of variables such as local film thickness and heat transfer have been made, techniques whereby these quantities can be measured over large areas are generally lacking. Point or average measurements may be appropriate at very low heat fluxes where insignificant evaporation occurs and the flow regime does not change, but such measurements are insufficient for model validation.

Techniques that have been used in the past to measure local film thickness have utilized capacitance sensors, conductance probes, confocal microscopes, and other techniques such as reflectance (e.g., Coney, 1973, Klausner et al., 1990, Han and Shikazono, 2009, and Shedd and Newell, 1998). The heat transfer has usually been measured using thermocouples welded to the tube walls or by resistively heating the walls and measuring the average wall temperature. The authors are not aware of any techniques whereby liquid film and heat transfer distributions in flow boiling are measured over relatively large areas with high resolution. If the heat transfer distribution along the walls of a tube could be measured, it could be used to verify models of the heat transfer variations predicted by models of slug flow, wavy annular flow, annular flow dryout, etc. For example, a three-zone model of elongated bubble

\footnotetext{
* Corresponding author. Tel.: +1 3014055437.

E-mail address: kimjh@umd.edu (J. Kim).
}

evaporation in microchannels was proposed by Thome et al. (2004) and Dupont et al. (2004). The model assumes that heat is transferred to a liquid slug, an elongated bubble, and a vapor slug (Fig. 1). Heat transfer to the elongated bubble is thought to occur through the thin liquid film between the vapor and the wall. The bubble frequency, liquid film thickness, and heat transfer to the liquid and vapor slugs are obtained from correlations. The globally averaged heat flux was measured and used for model verification. Much stronger verification of the model can be made if measurements of the local heat flux were available to verify the heat transfer rates before, during, and after passage of the bubble.

In flow regime based models (e.g., Kattan et al., 1998a,b,c), the local flow boiling heat transfer coefficients are predicted based on the tube perimeter fraction wetted by liquid. For example, for a horizontal stratified flow in a circular tube, the local heat transfer coefficient is given by

$h_{t p}=\frac{r_{i} \theta_{\text {dry }} h_{\text {vapor }}+r_{i}\left(2 \pi-\theta_{\text {dry }}\right) h_{\text {wet }}}{2 \pi r_{i}}$

where $h_{\text {wet }}$ is assumed to be composed of a nucleate boiling component and a convective component in a power law form:

$h_{w e t}=\left(h_{n b}^{3}+h_{c}^{3}\right)^{1 / 3}$

For annular and intermittent flow, $h_{t p}=h_{\text {wet }}$. The local void fraction needed to evaluate liquid and vapor velocities is calculated using correlations (e.g., Rouhani and Axelsson, 1970). Again, verification of these models can benefit greatly from measurements of the local heat transfer distribution in the various regimes. 


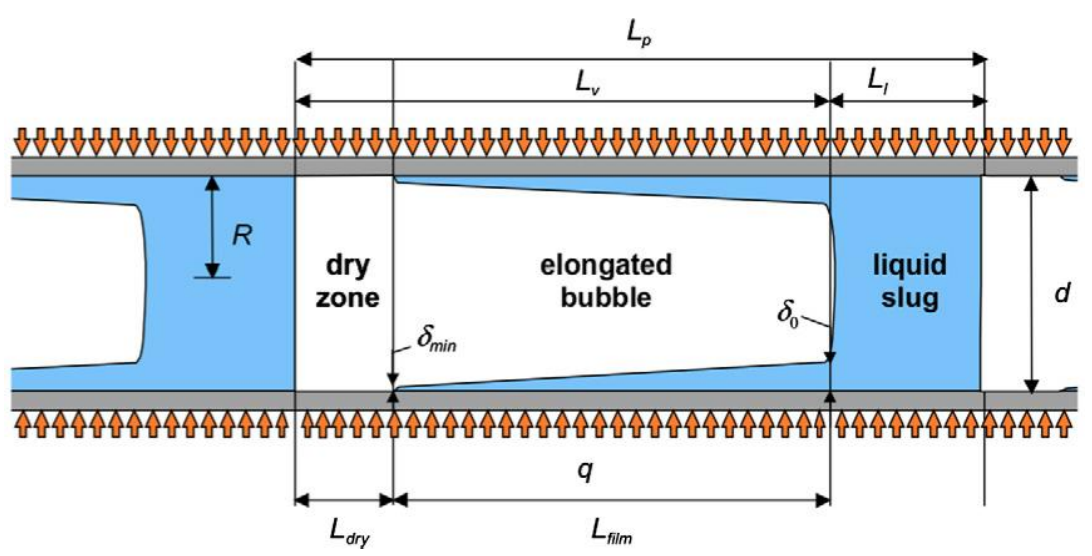

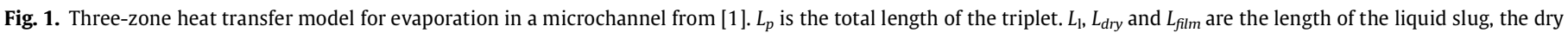

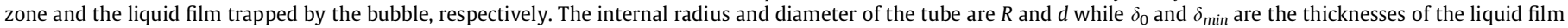
trapped between the elongated bubble and the channel wall at its formation and at dry out.

The objectives of this paper are to describe and demonstrate an IR thermometry based technique whereby the local heat transfer produced by two-phase flows can be measured with high spatial and temporal resolution. The application of the technique to the measurement of liquid film thickness is also described. IR thermometry is an established technology that can be used to measure temperatures when optical access to the surface is available in the wavelengths of interest, and has been used to measure heat transfer distributions during pool boiling heat transfer. Golobic et al. (2009) and Stephan and co-workers (e.g., Schweizer and Stephan, 2009) used an IR camera to measure the heat transfer distribution under single nucleating bubbles as they grew on a thin metal foils. Stephan has recently begun using a thicker CaF substrate in place of the thin film in order to increase the heat capacity of the substrate (Fischer et al., 2011) so it is more representative of real surfaces. Gerardi et al. (2010) used a high speed IR camera in conjunction with a video camera to measure bubble behavior on an ITO heated sapphire substrate. The IR camera measured the temperature distribution at the ITO surface, while a video camera was used to visualize the fluid behavior. Krebs et al. (2010), Shen et al. (2010) and Mani et al. (2012) used IR thermography to study flow boiling in microchannels, droplet evaporation, and jet impingement, respectively. In these studies, an IR camera was used to view through a silicon substrate to visualize in great detail the temperature distribution at the silicon/water interface. Sefiane et al. (2008) used IR thermography to visualize the spontaneously occurring hydrothermal waves within evaporating methanol, ethanol, and FC-72 droplets.

In the present work, a midwave IR camera (3.6-5.1 $\mu \mathrm{m})$ is used to measure the temperature variations within a multilayer consisting of a silicon substrate coated with a thin thermal insulator that is partially transparent to IR. The insulator amplifies the temperature variations and provides a strong signal for the IR camera. Since silicon is largely transparent to IR radiation, the temperature of the inner and outer walls of the multilayer can be measured by coating selected areas with a thin IR opaque film. The fluid used (FC-72) is also partially transparent to IR over a broad range of wavelengths, allowing the flow to be visualized and the film thickness to be measured. The theoretical basis for the technique, a description of the test apparatus and data reduction procedure, and experimental validation are presented in the sections below.

\section{Theoretical background}

Consider the multilayer wall consisting of a silicon substrate onto which polyimide tape (polyimide layer + acrylic adhesive) is attached as shown schematically on Fig. 2a. An opaque black paint that is much thinner than the other layers is applied to the top of the polyimide tape. The black surface is exposed to a two-phase flow. The polyimide tape is necessary to measure heat transfer coefficient distributions of the expected magnitude since the high thermal conductivity of the silicon substrate would simply smear out any temperature variations through substrate conduction, reducing both the magnitude of the temperature differences as well as the spatial resolution.

To obtain the heat transfer coefficient at the fluid-wall interface, the temperature gradient within the polyimide tape is required. If the time-varying temperatures of the black surface $T_{s 1}(t)$ and $T_{s 2}(t)$ are known, the instantaneous temperature profile within the multilayer can be obtained through an unsteady heat conduction simulation. If the polyimide tape is thin compared with the spatial resolution of the camera and the temperature gradient is much larger in the $x$-direction than in the $y$ - and $z$-directions, a 1 -D heat conduction assumption can be used. The 3-D heat conduction equation must be used otherwise. Assuming 1-D heat conduction, the governing equations within the layers are given by

$$
\begin{aligned}
& \rho_{s i} c_{p, s i} \frac{\partial T}{\partial t}=k_{s i} \nabla^{2} T+\dot{q}_{s i} \\
& \rho_{A} c_{p, A} \frac{\partial T}{\partial t}=k_{A} \nabla^{2} T \\
& \rho_{P} c_{p, P} \frac{\partial T}{\partial t}=k_{P} \nabla^{2} T
\end{aligned}
$$

and the system is subject to the boundary conditions $T=T_{s 1}(y, z, t)$ at $x=0, T=T_{s 2}(y, z, t)$ at $x=L_{S i}+L_{A}+L_{P}$.

Consider now the calculation of the black surface temperature $T_{s 1}(t)$. This temperature is not directly available since the energy measured by the IR camera consists of emission from the black surface, emission from each of the layers (which depends on the temperature profile within them), and reflection from the surroundings. Since the optical properties of the polyimide and adhesive are similar, they are treated as a single layer in the radiation calculation as indicated in Fig. 2b. The total energy measured by the camera $\left(E_{c}\right)$ is the sum of the energies emitted by each layer within the spectral bandwidth of the IR camera $\left(\lambda_{1}-\lambda_{2}\right)$ :

$E_{c}=\rho_{\infty-c} E_{\infty}+\varepsilon_{S i-c} E_{S i}+\varepsilon_{T-c} E_{T}+\tau_{s-c} E_{S}$

where $E_{\infty}=F_{\lambda_{1}-\lambda_{2}} \sigma T_{\infty}^{4}$ is the blackbody radiation due to the surroundings, $E_{S i}=\int_{0}^{L_{S i}} \alpha_{S i} F_{\lambda_{1}-\lambda_{2}} \sigma\left[T_{S i}(x)^{-4} \exp \left(-\alpha_{S i} x\right) d x\right.$ is the energy emitted by the silicon that reaches the $\mathrm{Si}-\infty$ interface, 

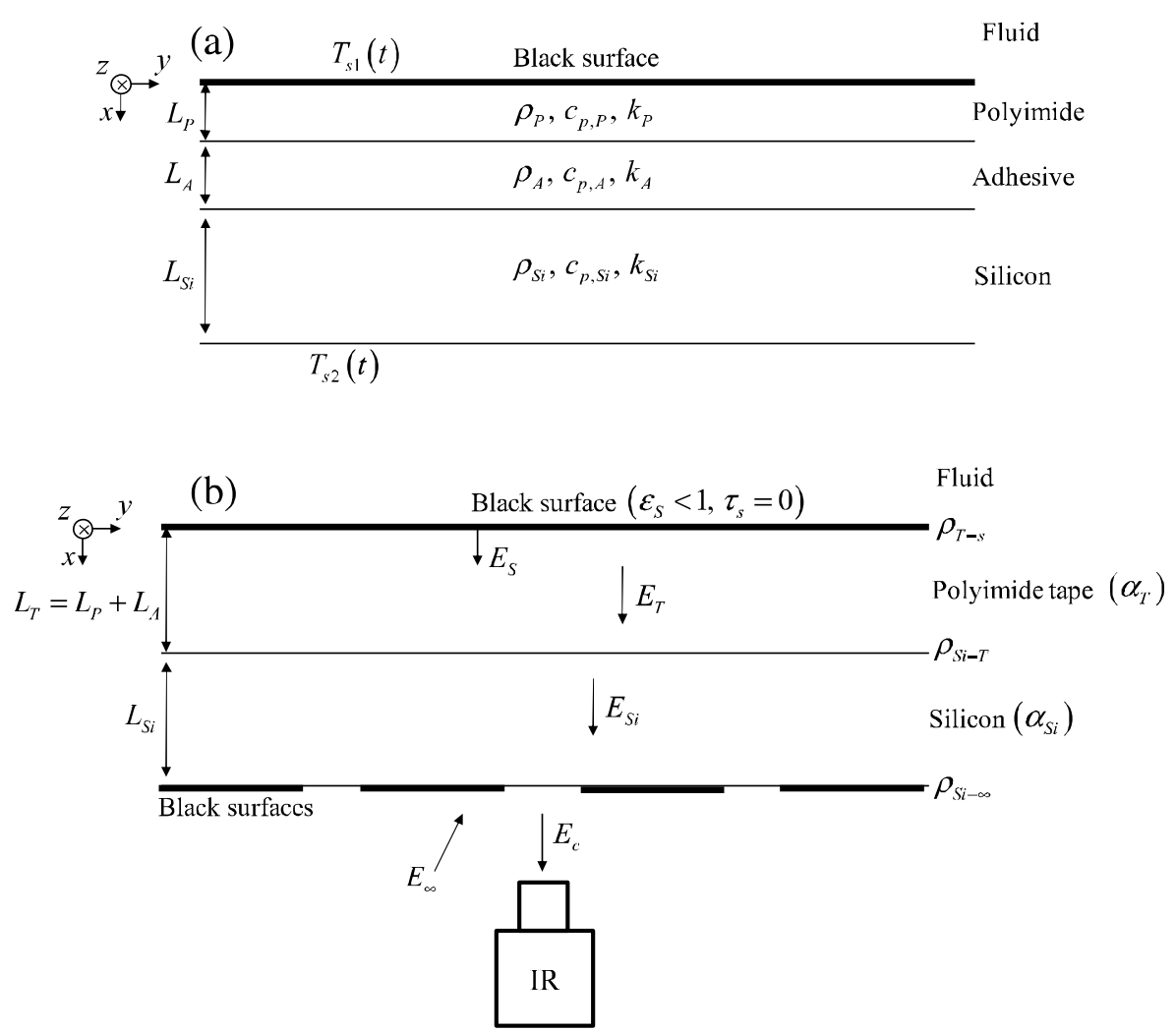

Fig. 2. Model descriptions. (a) System schematic for the conduction problem. (b) System schematic for the radiation problem.

$E_{T}=\int_{0}^{L_{T}} \alpha_{T} F_{\lambda_{1}-\lambda_{2}} \sigma\left[T_{T}(x)\right]^{4} \exp \left(-\alpha_{T} x\right) d x$ is the energy emitted by the tape that reaches the T-Si interface, $E_{s}=F_{\lambda_{1}-\lambda_{2}} \sigma T_{s, 1}^{4}$ is the blackbody radiation of the black surface.

$F_{\lambda_{1}-\lambda_{2}}$ is total emitted energy from a blackbody contained with the wavelength interval $\lambda_{1}-\lambda_{2}$ and can be obtained from tables or by integrating the Plank distribution. The coefficients $\rho_{\infty-c}, \varepsilon_{S i-c}$, $\varepsilon_{T-c}$, and $\tau_{s-c}$ account for the attenuation and reflection within the multilayer wall, and are given by

$\rho_{\infty i-c}=\rho_{S i-\infty}+\frac{\left(1-\rho_{S i-\infty}\right)^{2} \rho_{a p p, S i-T} \tau_{S i}^{2}}{1-\rho_{S i-\infty} \rho_{a p p, S i-T} \tau_{S i}^{2}}$

$\varepsilon_{S i-c}=\frac{\left(1-\rho_{S i_{-\infty}}\right)\left(1+\rho_{a p p, S i-T} \tau_{S i}\right)}{1-\rho_{S i-\infty} \rho_{a p p, S i-T} \tau_{S i}^{2}}$

$\varepsilon_{T-c}=\frac{\left(1-\rho_{S i-\infty}\right)\left(1-\rho_{S i-T}\right)\left(1+\rho_{T-S} \tau_{T}\right) \tau_{S i}}{\left(1-\rho_{S i-\infty} \rho_{a p p, S i-T} \tau_{S i}^{2}\right)\left(1-\rho_{S i-T} \rho_{T-S} \tau_{T}^{2}\right)}$

$\tau_{s-c}=\frac{\left(1-\rho_{S i_{-\infty}}\right)\left(1-\rho_{S i-T}\right)\left(1-\rho_{T-S}\right) \tau_{S i} \tau_{T}}{\left(1-\rho_{S i-\infty} \rho_{a p p, S i-T} \tau_{S i}^{2}\right)\left(1-\rho_{S i-T} \rho_{T-s} \tau_{T}^{2}\right)}$

$\rho_{a p p, S i-T}=\rho_{S i-T}+\frac{\left(1-\rho_{S i-T}\right)^{2} \rho_{T-S} \tau_{T}^{2}}{1-\rho_{S i-T} \rho_{T-s} \tau_{T}^{2}}$

The derivation of the above equations is given in the Appendix. The optical properties of the various layers along with the temperature of the surroundings $\left(T_{\infty}\right)$ are assumed to be known. The temperature distribution within the silicon $\left[T_{S i}(x)\right]$ and the tape $\left[T_{T}(x)\right]$ and the temperature of the black surface $\left(T_{s, 1}\right)$ are not known initially, but can be obtained by solving the coupled conduction and radiation problems according to the following algorithm:
1. Assume an arbitrary temperature profile within the multilayer at $t=0$.

2. Compute $E_{S i}$ and $E_{T}$ from the temperature distribution and determine an updated $E_{s}$ and surface temperature $T_{s, 1}$ from Eq. (4).

3. Solve the conduction equation using the updated $T_{s, 1}$ to obtain a new temperature profile at $t=\Delta t$.

4. Repeat steps 2 and 3 for each successive time step.

The effect of the assumed initial temperature profile within the multilayer will decay after which the true temperature profile will be known. ${ }^{1}$ The heat flux from the wall to the fluid can be obtained from the derivative of the instantaneous temperature profile within the polyimide at the black surface according to $q^{\prime \prime}=-\left.k_{P} \frac{\partial T}{\partial x}\right|_{x=0}$.

\section{Experimental results}

\subsection{Radiant property determination}

The above model requires knowledge of the reflectivity and absorptivity of the materials used. These were determined experimentally using the two configurations shown in Fig. 3. The blackbody consisted of a large cylindrical cavity at a controlled temperature behind a small orifice as shown in Fig. 4. The emissivity of this blackbody is estimated to be $>0.999$ using the relation provided in Quinn (1967).

The apparent reflectivity was determined using the experimental setup shown in Fig. 3a. The blackbody was positioned such that emission from it reflected from either the front or back surface of the layer to be tested. The total energy reaching the IR camera consisted of the blackbody energy reflected from the layer, the self-

\footnotetext{
${ }^{1}$ For the condition used for the experimental verification described below, the initial transient was found to have completely decayed after $0.1 \mathrm{~s}$.
} 
emission of the layer, and the transmission of energy from the surroundings through the layer:

$$
\begin{aligned}
E_{c 1}= & \rho_{a p p, m-\infty}\left(F_{\lambda_{1}-\lambda_{2}} \sigma T_{b}^{4}\right)+\varepsilon_{a p p, m-\infty}\left(F_{\lambda_{1}-\lambda_{2}} \sigma T_{m}^{4}\right) \\
& +\tau_{a p p, m-\infty}\left(F_{\lambda_{1}-\lambda_{2}} \sigma T_{\infty}^{4}\right)
\end{aligned}
$$

The measurement was then repeated without the blackbody:

$$
\begin{aligned}
E_{c 2}= & \rho_{a p p, m-\infty}\left(F_{\lambda_{1}-\lambda_{2}} \sigma T_{\infty}^{4}\right)+\varepsilon_{a p p, m-\infty}\left(F_{\lambda_{1}-\lambda_{2}} \sigma T_{m}^{4}\right) \\
& +\tau_{a p p, m-\infty}\left(F_{\lambda_{1}-\lambda_{2}} \sigma T_{\infty}^{4}\right)
\end{aligned}
$$

Subtraction of Eq. (10) from Eq. (11) allowed the apparent reflectivity to be determined since the temperature $T_{b}$ and $T_{\infty}$ are known.

The apparent transmissivity was determined using the setup shown in Fig. 3b. The total energy reaching the camera with and without the blackbody are given by

$$
\begin{aligned}
E_{c 3}= & \rho_{a p p, m-\infty}\left(F_{\lambda_{1}-\lambda_{2}} \sigma T_{\infty}^{4}\right)+\varepsilon_{a p p, m-\infty}\left(F_{\lambda_{1}-\lambda_{2}} \sigma T_{m}^{4}\right) \\
& +\tau_{a p p, m-\infty}\left(F_{\lambda_{1}-\lambda_{2}} \sigma T_{b}^{4}\right) \\
E_{c 4}= & \rho_{a p p, m-\infty}\left(F_{\lambda_{1}-\lambda_{2}} \sigma T_{\infty}^{4}\right)+\varepsilon_{a p p, m-\infty}\left(F_{\lambda_{1}-\lambda_{2}} \sigma T_{m}^{4}\right) \\
& +\tau_{a p p, m-\infty}\left(F_{\lambda_{1}-\lambda_{2}} \sigma T_{\infty}^{4}\right)
\end{aligned}
$$

Subtracting one from the other allowed the apparent transmissivity to be determined. The apparent reflectivity and transmissivity for a single layer $m$ sandwiched by two identical layers $n$ are given by

$\rho_{\text {app }, m-\infty}=\rho_{m-\infty}+\frac{\left(1-\rho_{m-\infty}\right)^{2} \rho_{m-\infty} \tau_{m}^{2}}{1-\rho_{m-\infty}^{2} \tau_{m}^{2}}$

$\tau_{a p p, m-\infty}=\frac{\left(1-\rho_{m-\infty}\right)^{2} \tau_{m}}{1-\rho_{m-\infty}^{2} \tau_{m}^{2}}$

(see Appendix for a derivation), from which $\rho_{m-\infty}$ and $\tau_{m}$ can be obtained. The absorption coefficient can then be obtained from the Beer-Lambert law, $\tau_{m}$, and the layer thickness.

The above technique was verified by measuring the reflectivity of a double side-polished silicon wafer $(n=3.43$ at $\lambda=3.7 \mu \mathrm{m})$ in air $(n=1.00)$. An analytical expression for the reflectivity at the silicon-air interface is given by

$\rho_{\text {Si-air }}=\left(\frac{n_{S i}-n_{\text {air }}}{n_{S i}+n_{\text {air }}}\right)^{2}=0.301$

This was in good agreement with the experimentally measured value of $\rho_{\text {Si-air }}=0.34$.

The reflectivity of the tape was calculated using the indices of refraction of the polyimide $\left(n_{\text {polyimide }}=1.7\right)$ and air to be

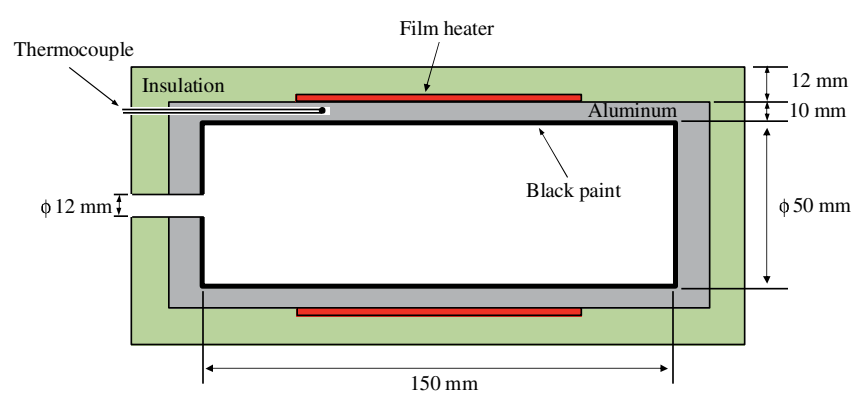

Fig. 4. Blackbody construction.

$\rho_{\text {polyimide-air }}=\left(\frac{n_{\text {polyimide }}-n_{\text {air }}}{n_{\text {polyimide }}+n_{\text {air }}}\right)^{2}=0.07$

If the index of refraction of the acrylic adhesive was assumed to be that of acrylic ( $n_{\text {adhesive }}=1.5$ ), the reflection would be $\rho_{\text {adhesive-air }}=0.04$. The reflection between the polyimide and adhesive is very small $\rho_{\text {polyimide-adhesive }}=0.004$. Since reflection within the tape is small and since the reflectivities of the polyimide and the acrylic adhesive are similar, the tape was considered to behave as a single optical layer with polyimide properties. The absorption coefficient of the tape was measured experimentally. A summary of the optical properties of the silicon and polyimide tape are given on Table 1. The absorption coefficient of the polyimide tape is seen to be much larger than that of the silicon.

\subsection{Uncertainty analysis}

The experimental uncertainty in the calculated heat flux was determined by perturbing each experimental parameter a small amount one at a time and running the algorithm to find the sensitivity to the variables at varying heat fluxes. Many of the assumed physical constants (e.g. thermal conductivity and thickness of silicon) introduced errors that were several orders of magnitude smaller than the important sources of error and were therefore omitted. The parameters that resulted in the highest error along with their values and assumed are tabulated in Table 2. The maximum total error, calculated as the RMS of each individual error, increases with heat flux and is summarized in Table 3 at various heat fluxes.

\subsection{Validation and demonstration}

\subsubsection{Air cooling}

To validate the experimental technique and algorithm, the apparatus shown in Fig. 5 was used. A Si wafer was heated by a thin film heater. Polyimide tape ( $30 \mu \mathrm{m}$ total thickness) with a thin black coating was deposited onto the top of the Si wafer to
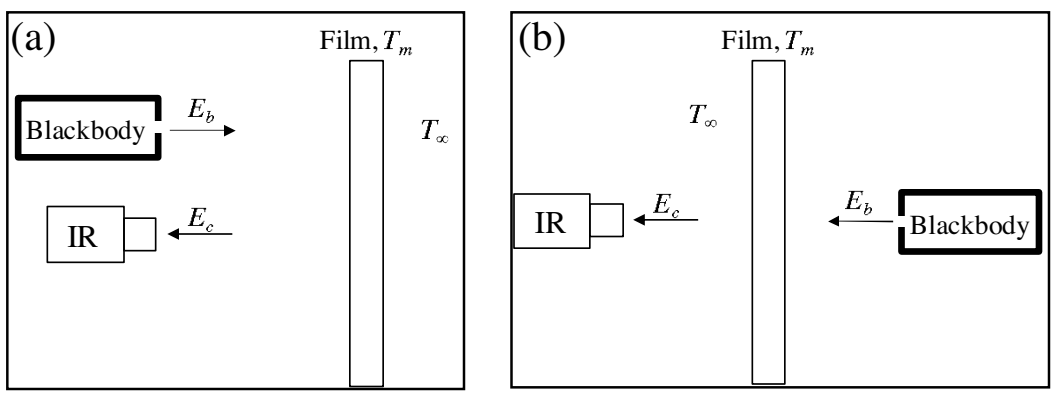

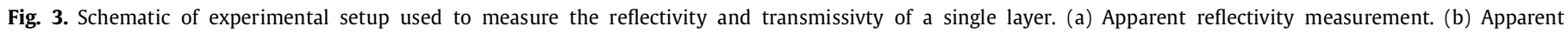
transmissivity measurement. 
Table 1

Optical properties of the silicon and polyimide tape.

\begin{tabular}{ll}
\hline Material & Absorption coefficient $\left(\mathrm{m}^{-1}\right)$ \\
Silicon & 52.6 \\
Polyimide tape & 7110 \\
Interface & Reflectivity \\
Si-air & 0.34 \\
Polyimide-air & 0.07 \\
Polyimide-Si & 0.14 \\
\hline
\end{tabular}

measure the top surface temperature $T_{s 1}(t)$. The black coating ( $6 \mu \mathrm{m}$ thick) was produced by silk screening onto the polyimide tape Nazdar GV111 paint consisting of about $20 \%$ carbon black in a vinyl chloride/vinyl acetate copolymer after curing. The high carbon black content ensured the coating was opaque, and its high thermal conductivity relative to the polyimide and thinness allowed its temperature to be assumed to be uniform.

The emissivity of the black surface was experimentally determined. The polyimide tape with black coating was attached to a heated aluminum plate containing a thermocouple. The plate was heated to temperatures between $45-95^{\circ} \mathrm{C}$, and the energy emitted from the surface along with the energy reflected from the surroundings were measured using the IR camera. The total energy measured by the IR camera is given by

$E_{c}=\varepsilon F_{\lambda_{1}-\lambda_{2}} \sigma T_{s}^{4}+(1-\varepsilon) F_{\lambda_{1}-\lambda_{2}} \sigma T_{\infty}^{4}$

from which the surface emissivity can be determined. The emissivity of the black coating was measured to be 0.90 .

A small piece of a polyimide tape with black coating was attached to the bottom of the wafer to measure the bottom surface temperature $T_{s 2}(t)$. Gold coated mirrors were used to simultaneously measure the energy emitted from both the top $\left[E_{T o p}(t)\right]$ and bottom $\left[E_{B o t, c}(t), E_{B o t, b}(t)\right]$ surfaces. Since the black surfaces were not transparent to IR, the black surface temperatures on the top $\left[T_{s 1}(t)\right]$ and bottom $\left[T_{s 2}(t)\right]$ surfaces could be directly obtained using the energies $\left[E_{T o p}(t), E_{B o t, b}(t)\right]$ using an IR camera (Electrophysics Silver $660 \mathrm{M}$ ). The black surface temperature on the top of the wafer $T_{s 1}(t)$ could also be calculated based on the algorithm using $E_{B o t, c}(t)$ and $T_{s 2}(t)$. To validate this calculation, the calculated top surface temperature was compared with the temperature obtained from $E_{\text {Top }}(t)$ as the top of the wafer was cooled using an air jet. Results are shown in Fig. 6. The air jet was turned on at $t=5.7 \mathrm{~s}$, resulting in a drop in the surface temperature. When the air jet was turned off at $t=10.9 \mathrm{~s}$, the temperature recovered. Good agreement within $0.5^{\circ} \mathrm{C}$ was seen between the measured and computed values, demonstrating that the algorithm can be used to accurately predict the true surface temperature.

\subsubsection{Droplet evaporation}

To further demonstrate the power of the technique, the apparatus shown in Fig. 5 was used to measure the wall heat flux during

Table 2

Parameters with largest effect on uncertainty analysis.

\begin{tabular}{lll}
\hline Experimental constant & Value & Uncertainty \\
Emissivity of the black coating & 0.90 & 0.01 \\
Absorptivity of the polyimide tape & $7110\left(\mathrm{~m}^{-1}\right)$ & $500\left(\mathrm{~m}^{-1}\right)$ \\
Polyimide thickness & $15 \mu \mathrm{m}$ & $2 \mu \mathrm{m}$ \\
Adhesive thickness & $15 \mu \mathrm{m}$ & $2 \mu \mathrm{m}$ \\
Reflectivity of silicon-air interface & 0.34 & 0.017 \\
Reflectivity of silicon-polyimide interface & 0.14 & 0.007 \\
Thermal conductivity of polyimide & $0.12 \mathrm{~W} / \mathrm{m}-\mathrm{K}$ & $0.01 \mathrm{~W} / \mathrm{m}-\mathrm{K}$ \\
Thermal conductivity of adhesive & $0.20 \mathrm{~W} / \mathrm{m}-\mathrm{K}$ & $0.01 \mathrm{~W} / \mathrm{m}-\mathrm{K}$ \\
\hline
\end{tabular}

Table 3

Summary of uncertainty analysis.

\begin{tabular}{llllll}
\hline Heat flux $\left(\mathrm{W} / \mathrm{cm}^{2}\right)$ & 2.0 & 4.0 & 6.0 & 8.0 & 10.0 \\
Max Error $\left(\mathrm{W} / \mathrm{cm}^{2}\right)$ & 0.35 & 0.47 & 0.62 & 0.80 & 0.98 \\
\hline
\end{tabular}

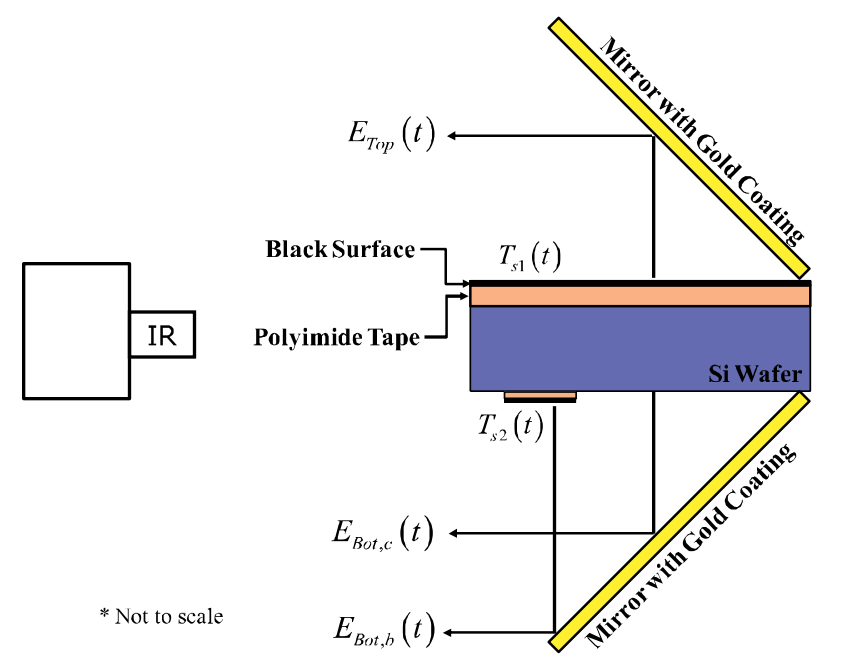

Fig. 5. Experimental setup for validation (not to scale).

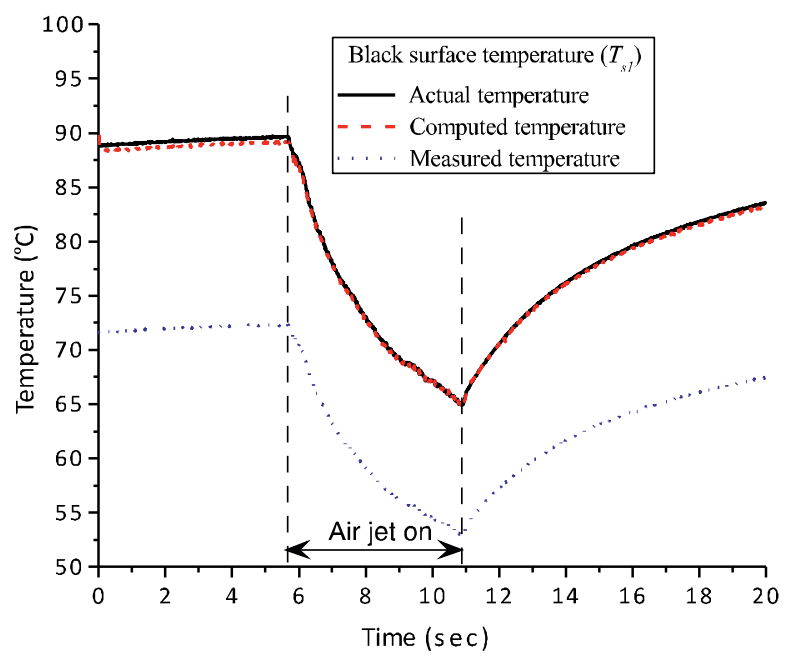

Fig. 6. Comparison of the top temperature, $T s_{1}(t)$, determined using the algorithm and the energy measured through the bottom $E_{B o t, c}(t)$ (computed temperature) with that measured directly using $E_{\text {top }}(t)$ (actual temperature).

evaporation of a single droplet of PF-5060. The substrate was heated to $83^{\circ} \mathrm{C}$ by attaching a thin film heater to the silicon wafer, after which a syringe was used to deposit a droplet onto the top surface. The IR camera was used to measure the heat transfer to the droplet from below the wafer. Fig. 7 shows temperature and heat flux contours as the droplet evaporates and illustrates how the local temperature and heat transfer can be measured with high spatial and temporal resolution. ${ }^{2}$

The droplet reached its first maximum diameter approximately $21 \mathrm{~ms}$ after impact, then contracted until $42 \mathrm{~ms}$. During this process, high heat transfer between the droplet and the heated surface was observed inside the droplet with a maximum heat transfer of

\footnotetext{
${ }^{2}$ A movie of the droplet evaporation process is available online at (mmc1.mpg).
} 

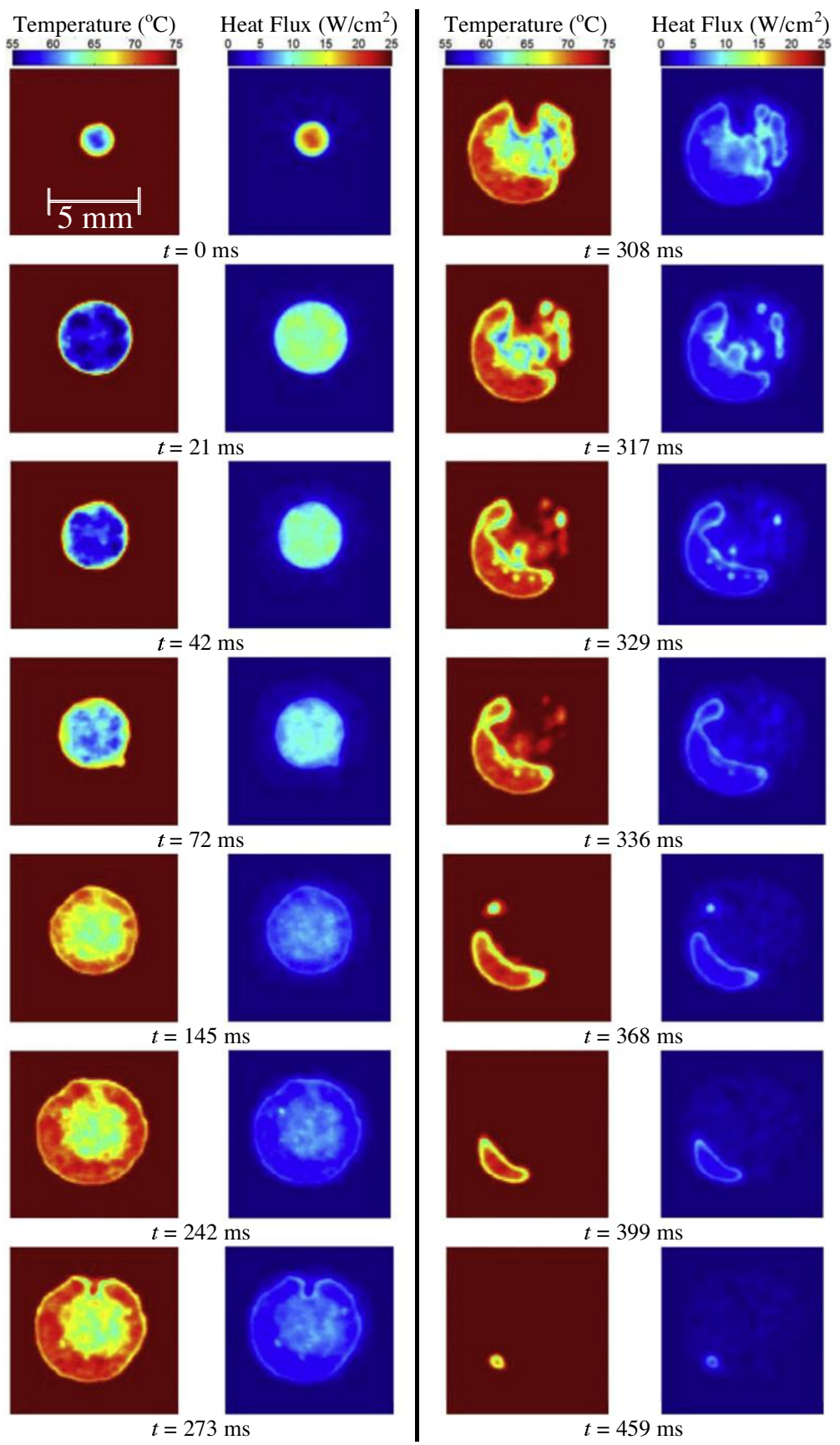

Fig. 7. Temperature and heat flux contours for droplet evaporation.

about $23 \mathrm{~W} / \mathrm{cm}^{2}$. After $42 \mathrm{~ms}$, the droplet spread again and the diameter reached a second maximum at $242 \mathrm{~ms}$. During this time, nucleate boiling within the droplet resulted in a higher heat transfer (about $10 \mathrm{~W} / \mathrm{cm}^{2}$ ) at the center of the droplet than at the edges. After $242 \mathrm{~ms}$ and until the droplet evaporates, the droplet size decreased due to evaporation at the rim of the droplet. Nucleate boiling ceased as the droplet thinned and ruptured. The highest heat transfer occurred at the receding three-phase contact line as expected.

The above results are for PF-5060 evaporating on the black painted surface and may not be representative for evaporation on silicon. Due to the highly wetting nature of PF-5060, however, the effect of contact angle is not expected to be important. Surface effects may be much larger when water is used, however.

\subsubsection{Gravity effects on flow boiling}

The technique was also used to measure flow boiling heat transfer during the high gravity and low gravity conditions produced by an aircraft. A polished tube $6 \mathrm{~mm} \mathrm{ID} \times 8 \mathrm{~mm}$ OD $\times 120 \mathrm{~mm}$ long was manufactured from a block of single crystal $n$-type silicon with $>1 \Omega$-cm resistivity. Two current taps and two voltage taps consisting of a chromium adhesion layer and $2000 \AA$ of aluminum were deposited at the ends of the tube after they were doped to minimize contact resistance $(<1 \Omega)$, allowing the power dissipated by the tube to be determined using a four-wire measurement. The tube was heated using a power supply that could impose up to $300 \mathrm{~V}$ across the tube, producing a nominal heat flux of up to $4 \mathrm{~W} / \mathrm{cm}^{2}$. Polyimide tape was attached to the inside of the tube, but only half of the tape had a black coating (Fig. 8). The mirror 


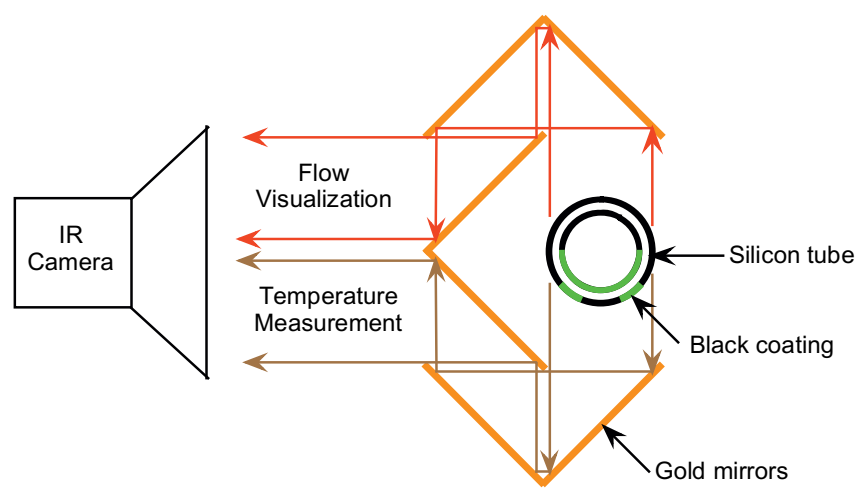

Fig. 8. Mirror setup to allow simultaneous flow visualization and measurement of multilayer temperatures. The green lines indicate the location of the black coating on the inner and outer surfaces of the silicon tube. (For interpretation of the references to color in this figure legend, the reader is referred to the web version of this article.)

arrangement allowed simultaneous visualization of the flow along with measurement of the temperature distribution on the inside and outside of the tube.

A flow loop (Fig. 9) was constructed so FC-72 could be pumped through the tube at known mass fluxes up to $200 \mathrm{~kg} / \mathrm{m}^{2}$-s and inlet temperatures from room temperature to near saturated $\left(T_{\text {sat }}=57^{\circ} \mathrm{C}\right.$ for FC-72 at $\left.1 \mathrm{~atm}\right)$. The primary loop included the silicon test section where wall temperature measurements were made using the IR camera. The two-phase mixture exiting the test section was condensed in a counterflow heat exchanger then pumped through a flowmeter and preheater before entering the test section again. An accumulator (expansion tank) was used to keep the pressure within the loop at nominally $1 \mathrm{~atm}$. The temperature of the FC-72 exiting the heat exchanger was controlled by occasionally pumping cold water through the heat exchanger.

The variable gravity environment was produced by a Zero G Corporation 727 aircraft flying parabolic arcs as part of NASA's FAST program in July, 2011. Approximately $20 \mathrm{~s}$ of $1.8 \mathrm{~g}$ pullup was followed by $20 \mathrm{~s}$ of low-g $( \pm 0.01 \mathrm{~g})$ and $20 \mathrm{~s}$ of $1.8 \mathrm{~g}$ pullout. Up to 40 parabolas were flown per flight and four flights were made. The test section was oriented such that the liquid entered the tube in a vertical upward flow configuration-because the flow produced is nominally axisymmetric, heat transfer data was reduced only along the axis of the tube. This configuration also avoids refraction effects that can occur due to the curvature of the tube when viewing the tube off-axis.

The heat transfer coefficient distribution is shown on Fig. 10a as the fluid flows through the tube in a low-g environment at a relatively low mass flux of $44 \mathrm{~kg} / \mathrm{m}^{2}$-s. ${ }^{3}$ Large variations are observed where liquid rewets the surface with peaks up to $12,000 \mathrm{~W} / \mathrm{m}^{2}-\mathrm{K}$. Very low heat transfer is also observed on the tube, indicating dryout of the surface. Time averaged heat transfer coefficient distributions along the tube in high-g and low-g are shown on Fig. 10b. The effect of gravity is minimal in the first half of the tube where the flow is dominated by bubbles dispersed in a liquid. The heat transfer deteriorates towards the tube exit, however, as the flow transitions to a wavy annular type flow with periodic dryout. Additional data at various flow rates and inlet enthalpies will be presented in future work.

\subsubsection{Liquid film thickness}

We close this paper with a discussion on how the technique can be used to measure liquid film thickness in a tube. Consider the case where a liquid film flows along the inside walls of a round

\footnotetext{
${ }^{3}$ A movie of flow boiling in low-g is available online at (mmc2.mp4).
}

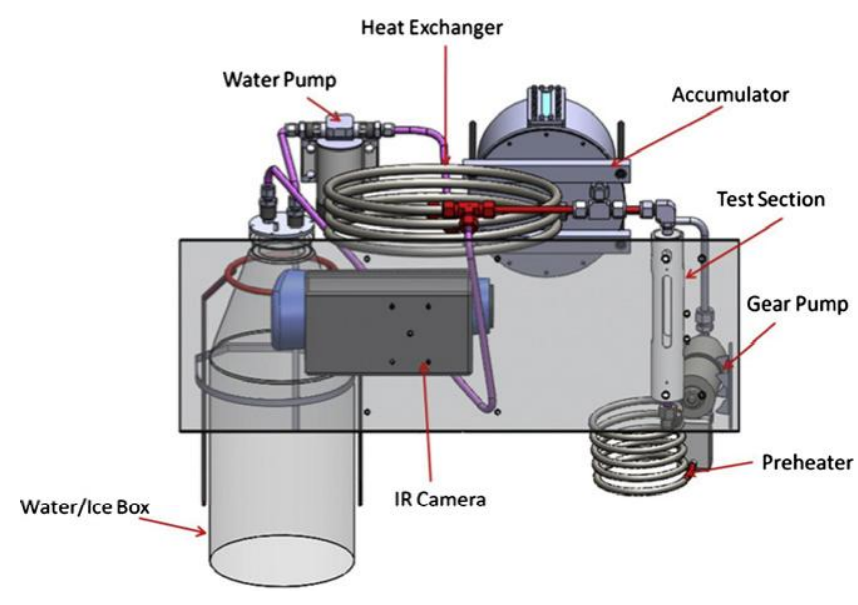

Fig. 9. Solid model of flow loop.

tube as can occur during annular flow (Fig. 11). The tube and liquid are in thermal equilibrium at temperature $T_{s}$, and placed in a large enclosure at temperature $T_{\infty}$. The IR camera looks through the tube at a black surface at $T_{\text {hot }}$. The energy measured by the IR camera $E_{c}$ consists of energy from the surroundings that are reflected from the front, the emission from the tube and liquid, and the energy emitted from $T_{\text {hot }}$ that is transmitted through the tube and liquid. It can be shown that

$$
\begin{aligned}
E_{c}= & \rho_{\infty f-c} E_{b, \infty}+\left[\left(1-\tau_{m}\right) \varepsilon_{m f-c}+\left(1-\tau_{l}\right) \varepsilon_{l f-c}+\left(1-\tau_{l}\right) \varepsilon_{l r-c}\right. \\
& \left.+\left(1-\tau_{m}\right) \varepsilon_{m r-c}\right] E_{b, S}+\tau_{h o t, r-c} E_{b, h o t}
\end{aligned}
$$

where

$$
\begin{aligned}
& E_{b, \infty}=\sigma T_{\infty}^{4}, \quad E_{b, S}=\sigma T_{S}^{4}, \quad E_{b, h o t}=\sigma T_{h o t}^{4} \\
& \tau_{l}=\exp \left(-\alpha_{l} L_{l}\right), \quad \tau_{m}-\exp \left(-\alpha_{m} L_{m}\right) \\
& \rho_{\propto f-c}=\rho_{\infty-m}+\frac{\left(1-\rho_{\infty-m}\right)^{2} \rho_{a p p, 4} \tau_{m}^{2}}{1-\rho_{\infty-m} \rho_{a p p, 4} \tau_{m}^{2}} \\
& \varepsilon_{m f-c}=\frac{\left(1-\rho_{\infty-m}\right)\left(1+\rho_{a p p, 4} \tau_{m}\right)}{1-\rho_{\infty-m} \rho_{a p p, 4} \tau_{m}^{2}} \\
& \varepsilon_{l f-c}=\frac{\left(1-\rho_{\infty-m}\right) \tau_{m}}{1-\rho_{\infty-m} \rho_{a p p, 4} \tau_{m}^{2}} \times \frac{\left(1-\rho_{l-m}\right)\left(1+\rho_{a p p, 3} \tau_{l}\right)}{1-\rho_{l-m} \rho_{a p p, 3} \tau_{l}^{2}} \\
& \varepsilon_{l r-c}=\frac{\left(1-\rho_{\infty-m}\right) \tau_{m}}{1-\rho_{\infty-m} \rho_{a p p, 4} \tau_{m}^{2}} \times \frac{\left(1-\rho_{l-m}\right) \tau_{l}}{1-\rho_{l-m} \rho_{a p p, 3} \tau_{l}^{2}} \times \frac{\left(1-\rho_{l-v}\right)}{1-\rho_{l-v} \rho_{a p p, 2}} \\
& \times \frac{\left(1-\rho_{l-v}\right)\left(1+\rho_{a p p, 1} \tau_{l}\right)}{1-\rho_{l-v} \rho_{a p p, 1} \tau_{l}^{2}} \\
& \varepsilon_{m r-c}=\frac{\left(1-\rho_{\infty-m}\right) \tau_{m}}{1-\rho_{\infty-m} \rho_{a p p, 4} \tau_{m}^{2}} \times \frac{\left(1-\rho_{l-m}\right) \tau_{l}}{1-\rho_{l-m} \rho_{a p p, 3} \tau_{l}^{2}} \times \frac{\left(1-\rho_{l-v}\right)}{1-\rho_{l-v} \rho_{a p p, 2}} \\
& \times \frac{\left(1-\rho_{l-v}\right)\left(1-\rho_{l-m}\right)\left(1+\rho_{\infty-m} \tau_{m}\right) \tau_{l}}{\left(1-\rho_{l-v} \rho_{a p p, 1} \tau_{l}^{2}\right)\left(1-\rho_{l-m} \rho_{\infty-m} \tau_{m}^{2}\right)} \\
& \tau_{h o t, r-c}=\frac{\left(1-\rho_{\infty-m}\right) \tau_{m}}{1-\rho_{\infty-m} \rho_{a p p, 4} \tau_{m}^{2}} \times \frac{\left(1-\rho_{l-m}\right) \tau_{l}}{1-\rho_{l-m} \rho_{a p p, 3} \tau_{l}^{2}} \times \frac{\left(1-\rho_{l-v}\right)}{1-\rho_{l-v} \rho_{a p p, 2}} \\
& \times \frac{\left(1-\rho_{l-v}\right)\left(1-\rho_{l-m}\right)\left(1-\rho_{\infty-m}\right) \tau_{l} \tau_{m}}{\left(1-\rho_{l-v} \rho_{a p p, 1} \tau_{l}^{2}\right)\left(1-\rho_{l-m} \rho_{\infty-m} \tau_{m}^{2}\right)}
\end{aligned}
$$$$
\rho_{a p p, 1}=\rho_{l-m}+\frac{\left(1-\rho_{l-m}\right)^{2} \rho_{\infty-m} \tau_{m}^{2}}{1-\rho_{l-m} \rho_{\infty-m} \tau_{m}^{2}}
$$ 


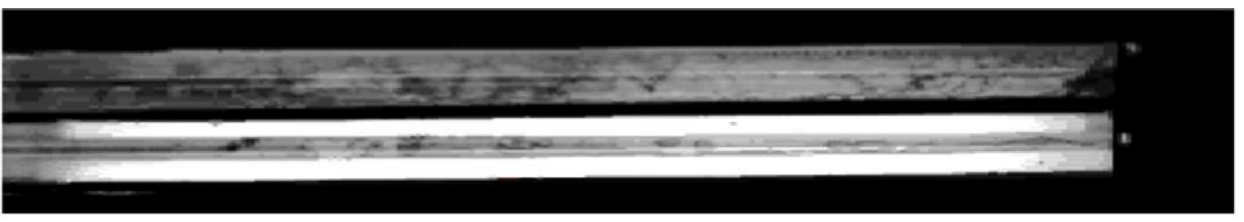

Time $=35.475 \mathrm{sec}$
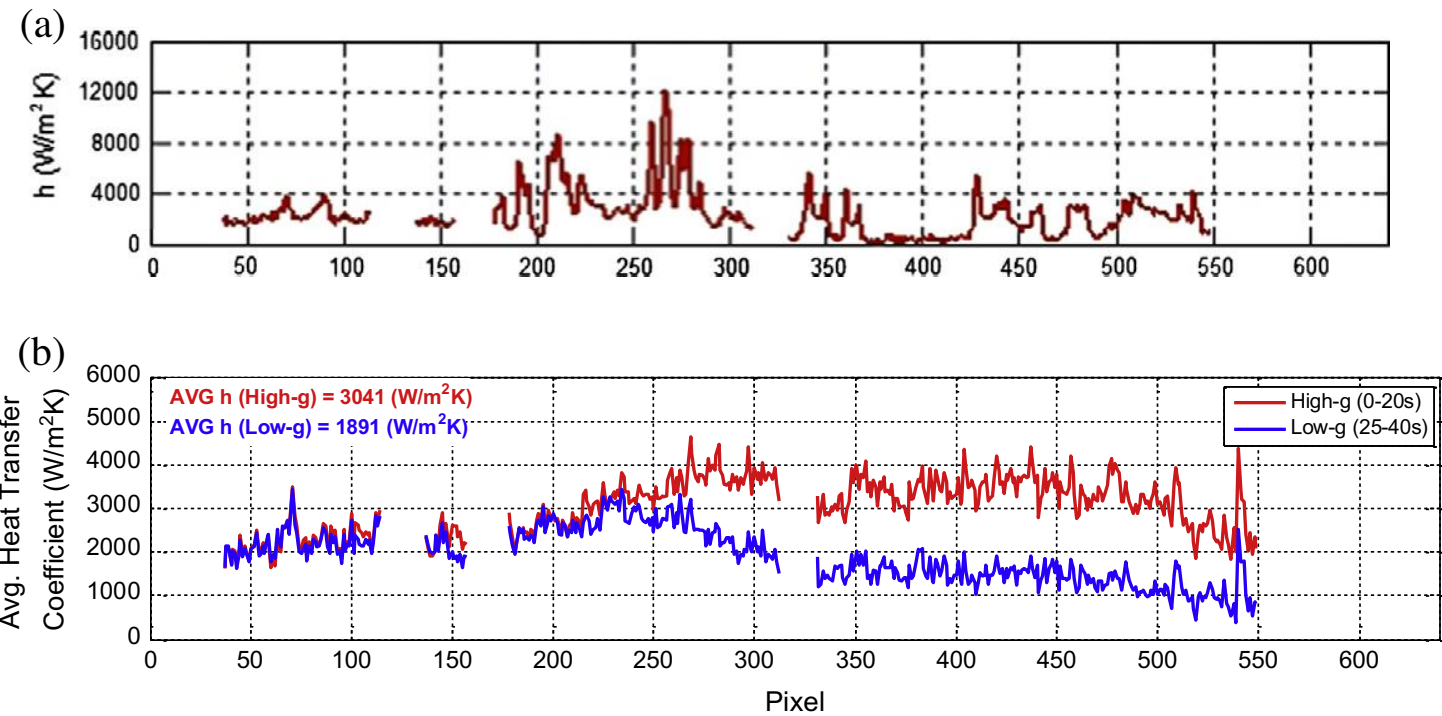

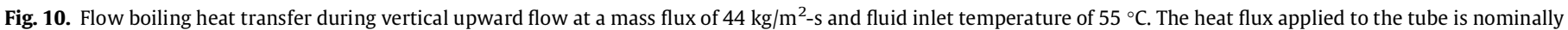

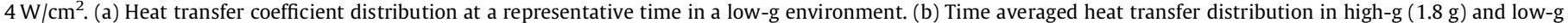
environments.

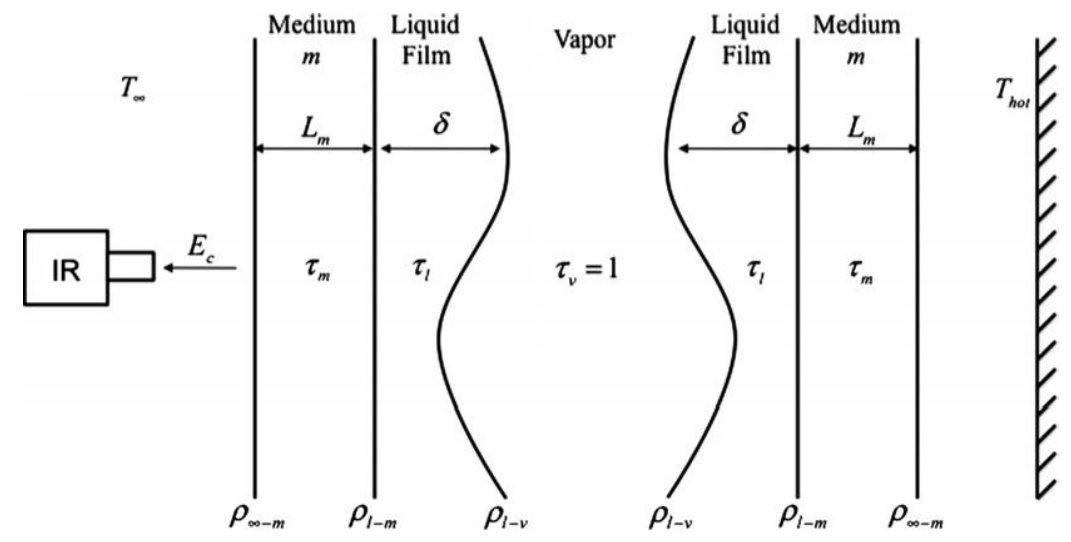

Fig. 11. Schematic of axisymmetric liquid layer flowing on the inside of a tube (medium $\mathrm{m}$ ).

$\rho_{a p p, 2}=\rho_{l-v}+\frac{\left(1-\rho_{l-v}\right)^{2} \rho_{a p p, 1} \tau_{l}^{2}}{1-\rho_{l-v} \rho_{a p p, 1} \tau_{l}^{2}}$

$\rho_{a p p, 3}=\rho_{l-v}+\frac{\left(1-\rho_{l-v}\right)^{2} \rho_{a p p, 2}}{1-\rho_{l-v} \rho_{a p p, 2}}$

$\rho_{a p p, 4}=\rho_{l-m}+\frac{\left(1-\rho_{l-m}\right)^{2} \rho_{a p p, 3} \tau_{l}^{2}}{1-\rho_{l-m} \rho_{a p p, 3} \tau_{l}^{2}}$

All parameters in Eq. (19) can be measured with the exception of $\tau_{l}$, from which the liquid film thickness $L_{l}$ can be determined. If heat transfer occurs across the liquid film, the temperature variation within the liquid needs to be accounted for.
The range of film thicknesses that can be measured depends primarily on the absorption coefficient of the fluid. A plot of the effective transmissivity of a liquid film flowing within a silicon tube (Fig. 11) with $1 \mathrm{~mm}$ thick walls as the liquid film thickness is varied is shown in Fig. 12. The effective transmissivity is only about 0.33 even when no liquid flows in the tube due to reflection at the silicon interfaces. As the liquid film thickness increases, the effective transmissivity decreases and the system becomes effectively opaque at large thicknesses. If it is assumed that the transmissivity can be accurately measured between $20 \%$ and $80 \%$ of these limits (horizontal dashed lines in Fig. 12), the range of liquid film thickness that can be measured can be found. For example, it is expected that only thin water films $(\sim 4-40 \mu \mathrm{m})$ can be measured due to its high absorption coefficient while FC-72 films can be measured over a much broader range $(\sim 150-1000 \mu \mathrm{m})$. 


\section{Closing remarks}

A new technique for the measurement of high resolution heat transfer distributions due to two phase flow phenomena based on IR thermometry has been described. The technique was demonstrated on droplet evaporation and flow boiling in low-g environments. The equations needed to calculate the thickness of an isothermal liquid film in round tubes were derived and estimates were made of liquid film thicknesses that can be measured.

\section{Acknowledgements}

This work was sponsored by NASA through Grant NNX09AK39A. The grant monitor was Mr. John McQuillen. The flights on the Zero G Corporation aircraft were made possible through the NASA FAST program.

\section{Appendix A}

\section{A.1. Beer-Lambert law}

Consider radiation of intensity $I_{0}\left(\mathrm{~W} / \mathrm{m}^{2}\right)$ striking a layer of thickness $L$. The intensity of the radiation $(I)$ exiting the other side is given by $I=I_{0} \exp (-\alpha L)$ where $\alpha\left(\mathrm{m}^{-1}\right)$ is the absorption coefficient and represents the ability of the layer to attenuate the incoming radiation. The transmissivity of a layer due to absorption alone can be expressed as $\tau=\exp (-\alpha L)$.

\section{A.2. Emission from translucent materials}

Translucent materials simultaneously generate and absorb radiation. Radiation generated within the layer must travel through the material before it reaches the surface, during which it is attenuated in accordance with the Beer-Lambert law. Part of the radiation reaching the surface is emitted and the remainder can be reflected back into the layer where it can be re-absorbed and re-reflected. The effective radiant properties of translucent materials is derived below. It is assumed that the values all optical properties are independent of temperature.

Consider first the radiation emitted by a differential layer of thickness $d x$ with an emission coefficient $\varepsilon\left(\mathrm{m}^{-1}\right)$ and an absorption coefficient $\alpha\left(\mathrm{m}^{-1}\right)$ as shown in Fig. A1. The emission coefficient characterizes how black the layer is. The radiation flux emitted from the differential thickness $d x$ in the $x$ direction is given by

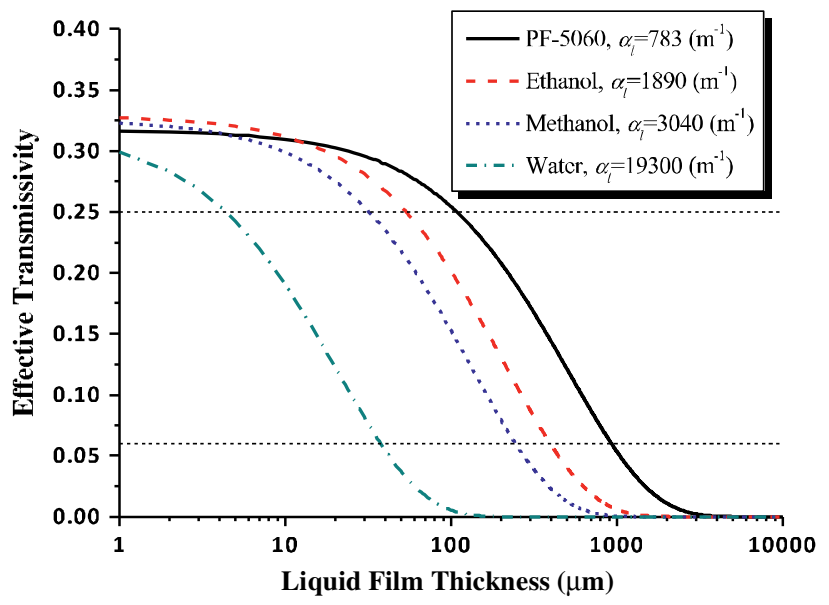

Fig. 12. Range of liquid film thicknesses that can accurately be measured on a silicon tube. $\varepsilon \sigma[T(x)]^{4} d x$. Because the differential element is thin, all of this radiation leaves the differential volume. The radiation that is not absorbed by the material that strikes the surface of the film at $x=0$ is given by $\varepsilon \sigma[T(x)]^{4} \exp [-\alpha x] d x$. The total radiation energy generated by the layer that strikes $x=0$ can then be obtained by integration:

$E=\int_{0}^{L} \varepsilon \sigma[T(x)]^{4} \exp [-\alpha x] d x$

For the special case where the temperature in the layer is uniform, Eq. (A.1) reduces to

$E=\frac{\varepsilon}{\alpha} \sigma T^{4}[1-\exp (-\alpha L)]$

In the limit of infinitely thick films, Eq. (A.2) indicates that the energy flux is

$E_{L \rightarrow \infty}=\frac{\varepsilon}{\alpha} \sigma T^{4}$

Since very thick films act like a blackbody radiating energy at $T$, the layer will emit radiation according to $E_{L \rightarrow \infty}=\sigma T^{4}$, indicating that the relation between emission and absorption coefficients is $\varepsilon=\alpha$.

Consider next reflection at the surfaces. As mentioned above, part of the radiation reaching the surface of a layer can be reflected at the surface or transmitted through the surface multiple times as shown in Fig. A2 where medium $m$ is sandwiched between two other media $\infty$ and $n$. The energy emitted by the layer that reaches the $\propto-m$ interface is given by $E_{m}$ and can be calculated from Eq. (A.1). The fraction of this energy that escapes from this surface is $\left(1-\rho_{\infty-m}\right) E_{m}$ while the energy reflected back into $m$ is $\rho_{\infty-m} E_{m}$. The reflected energy that is not absorbed within $m$ before arriving at the $m-n$ interface is given by $\exp \left(-\alpha_{m} L_{m}\right) \rho_{\infty-m} E_{m}=\tau_{m} \rho_{\infty-m} E_{m}$. This radiation is reflected again at the $m-n$ interface, absorbed within $m$, and arrives at the $\infty-m$ interface. The second contribution to the energy that escapes the $\infty-m$ interface is thus

$\left(1-\rho_{\infty-m}\right) \rho_{\infty-m} \rho_{m-n} \tau_{m}^{2} E_{m}$

This process of reflection occurs again with the result that the third contribution is

$$
\left(1-\rho_{\infty-m}\right) \rho_{\infty-m}^{2} \rho_{m-n}^{2} \tau_{m}^{4} E_{m}
$$

The energy escaping through the $\propto-m$ interface is therefore

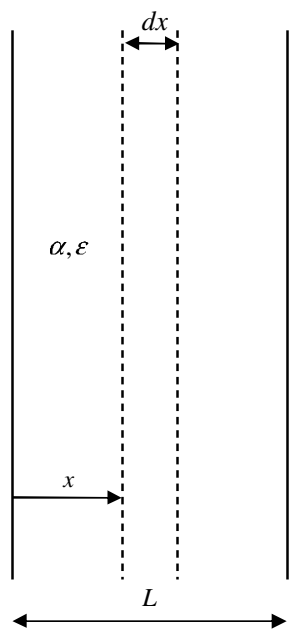

Fig. A1. Radiation emitted within a translucent layer. 


$$
\begin{aligned}
& \left(1-\rho_{\infty-m}\right) E_{m}+\left(1-\rho_{\propto-m}\right) \rho_{\infty-m} \rho_{m-n} \tau_{m}^{2} E_{m}+(1 \\
& \left.\quad-\rho_{\infty-m}\right) \rho_{\infty-m}^{2} \rho_{m-n}^{2} \tau_{m}^{4} E_{m}+\cdots \\
& \quad=\left(1-\rho_{\infty-m}\right) E_{m} \sum_{n=0}^{\infty}\left(\rho_{\infty-m} \rho_{m-n} \tau_{m}^{2}\right)^{n}
\end{aligned}
$$

Thus far, only the radiation emitted toward the $\propto-m$ interface has been considered. Radiation is also emitted toward the $m-n$ interface and part of this energy escapes through the $\infty-m$ interface. By applying the same processes as shown in Fig. A2b, the energy leaving the left surface can be expressed as.

$$
\begin{aligned}
& \left(1-\rho_{\infty-m}\right) \rho_{m-n} \tau_{m} E_{m}+\left(1-\rho_{\infty-m}\right) \rho_{m-n}^{2} \rho_{\infty-m} \tau_{m}^{3} E_{m}+(1 \\
& \left.\quad-\rho_{\infty-m}\right) \rho_{m-n}^{3} \rho_{\infty-m}^{2} \tau_{m}^{5} E_{m}+\cdots \\
& \quad=\left(1-\rho_{\infty-m}\right) \rho_{m-n} \tau_{m} E_{m} \sum_{n=0}^{\infty}\left(\rho_{\infty-m} \rho_{m-n} \tau_{m}^{2}\right)^{n}
\end{aligned}
$$

The total emission from the $\propto-m$ interface $\left(E_{m-\infty}\right)$ is the summation of Eqs. (A.6) and (A.7):

$$
E_{m-\infty}=\left(1-\rho_{\infty-m}\right)\left(1+\rho_{m-n} \tau_{m}\right) E_{m} \sum_{n=0}^{\infty}\left(\rho_{\infty-m} \rho_{m-n} \tau_{m}^{2}\right)^{n}
$$

Since $\rho_{\infty-m}, \rho_{m-n}$, and $\tau_{m}$ are always less than unity, the infinite series may be written in analytical form as

$E_{m-\infty}=\frac{\left(1-\rho_{\infty-m}\right)\left(1+\rho_{m-n} \tau_{m}\right)}{1-\rho_{\infty-m} \rho_{m-n} \tau_{m}^{2}} E_{m}$

Similarly, the energy emitted by $m$ that escapes from the $m-n$ interface $\left(E_{m-n}\right)$ is given by

$E_{m-n}=\frac{\left(1-\rho_{m-n}\right)\left(1+\rho_{\infty-m} \tau_{m}\right)}{1-\rho_{\infty-m} \rho_{m-n} \tau_{m}^{2}} E_{m}$

\section{A.3. Apparent reflectivity and apparent transmissivity of a single layer}

Let us first consider the reflection of incoming radiation $E_{\infty}$ from a single layer as shown in Fig. A3a. Part of the incoming energy is reflected from the $\infty-m$ interface. The remainder is attenuated by medium $m$, and part of this is reflected from the $m-n$ interface back towards the $\infty-m$ interface. Using a similar line of reasoning as above to account the infinite number of reflections, the total energy reflected from $m$ is the sum of the energies leaving the $\infty-m$ interface shown in Fig. A3a:

$$
\begin{aligned}
E_{\infty-m}= & \rho_{\infty-m} E_{\infty}+\left(1-\rho_{\infty-m}\right)^{2} \rho_{m-n} \tau_{m}^{2} E_{\infty}+(1 \\
& \left.-\rho_{\infty-m}\right)^{2} \rho_{\infty-m} \rho_{m-n}^{2} \tau_{m}^{4} E_{\infty}+\{1 \\
& \left.-\rho_{\infty-m}\right)^{2} \rho_{\infty-m}^{2} \rho_{m-n}^{3} \tau_{m}^{6} E_{\infty}+\cdots \\
- & E_{\infty}\left[\rho_{\infty-m}+\left(1-\rho_{\infty-m}\right)^{2} \rho_{m-n} \tau_{m}^{2}\right. \\
& \left.\times \sum_{n=0}^{\infty}\left(\rho_{\infty-m} \rho_{m-n} \tau_{m}^{2}\right)^{n}\right] \\
= & E_{\infty}\left[\rho_{\infty-m}+\frac{\left(1-\rho_{\infty-m}\right)^{2} \rho_{m-n} \tau_{m}^{2}}{1-\rho_{\infty-m} \rho_{m-n} \tau_{m}^{2}}\right]
\end{aligned}
$$

The apparent reflectivity is therefore given by

$\rho_{a p p, \infty-m}=\rho_{\infty-m}+\frac{\left(1-\rho_{\infty-m}\right)^{2} \rho_{m-n} \tau_{m}^{2}}{1-\rho_{\infty-m} \rho_{m-n} \tau_{m}^{2}}$

The apparent transmissivity of medium $m$ to $E_{\infty}$ is found in a similar manner by summing the energies leaving the $m-n$ interface in Fig. A3b:

$\tau_{a p p, \infty-m}=\frac{\left(1-\rho_{\infty-m}\right)\left(1-\rho_{m-n}\right) \tau_{m}}{1-\rho_{\infty-m} \rho_{m-n} \tau_{m}^{2}}$

The apparent transmissivity is seen to be independent of the direction of the incoming radiation.

The apparent absorptivity of $m$ can also be determined from a similar accounting of energy absorbed within $m$ to be

$\alpha_{a p p, \infty-m}=\frac{\left(1-\rho_{\infty-m}\right)\left(1+\rho_{m-n} \tau_{m}\right)\left(1-\tau_{m}\right)}{1-\rho_{m-n} \rho_{\infty-m} \tau_{m}^{2}}$

We leave it as an exercise to the reader to verify that $\alpha_{a p p, \infty-m}+\rho_{a p p, \infty-m}+\tau_{a p p, \infty-m}=1$. These apparent properties are the same as those presented by McMahon (1950) if $\rho_{\infty-m}=\rho_{m-n}$ and the temperature distribution in medium $m$ is uniform.
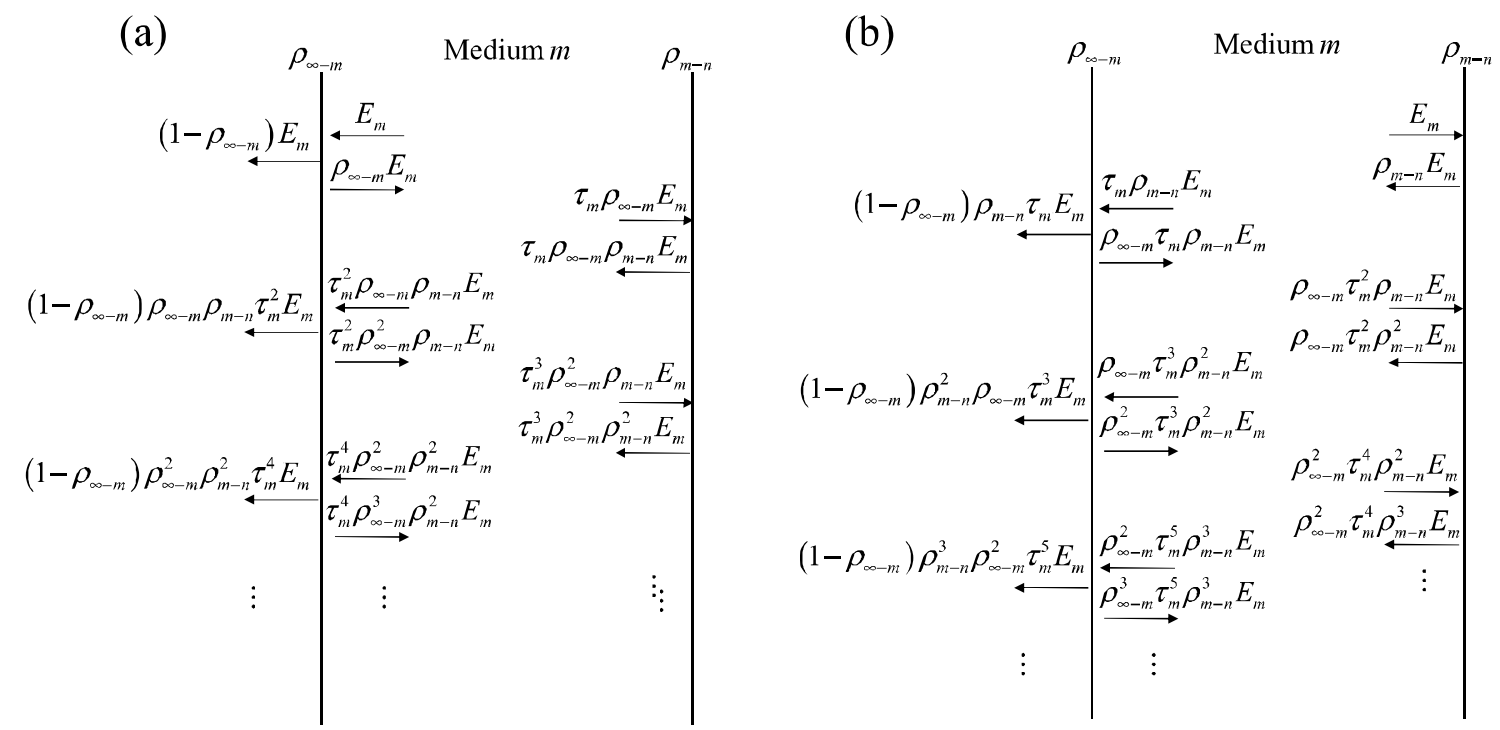

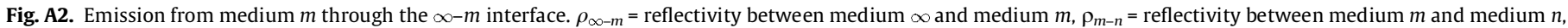

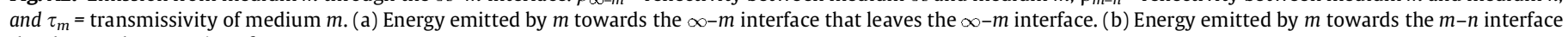
that leaves the $\infty-m$ interface. 
(a)

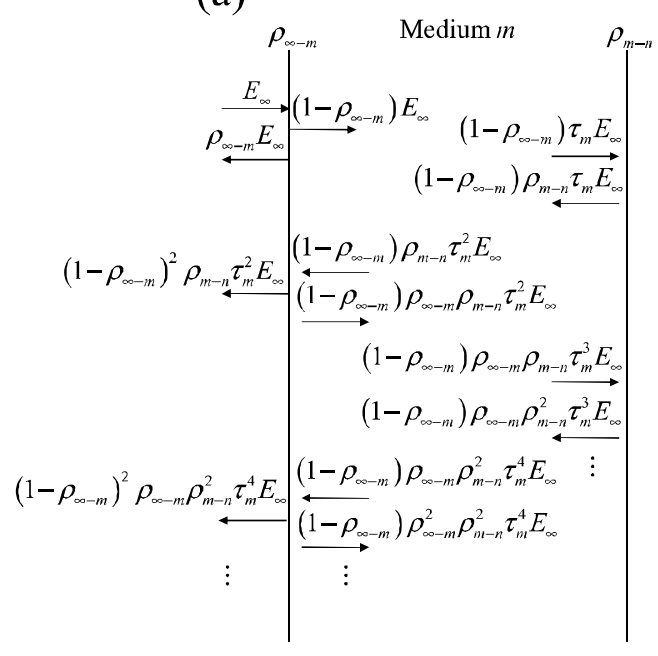

(b)

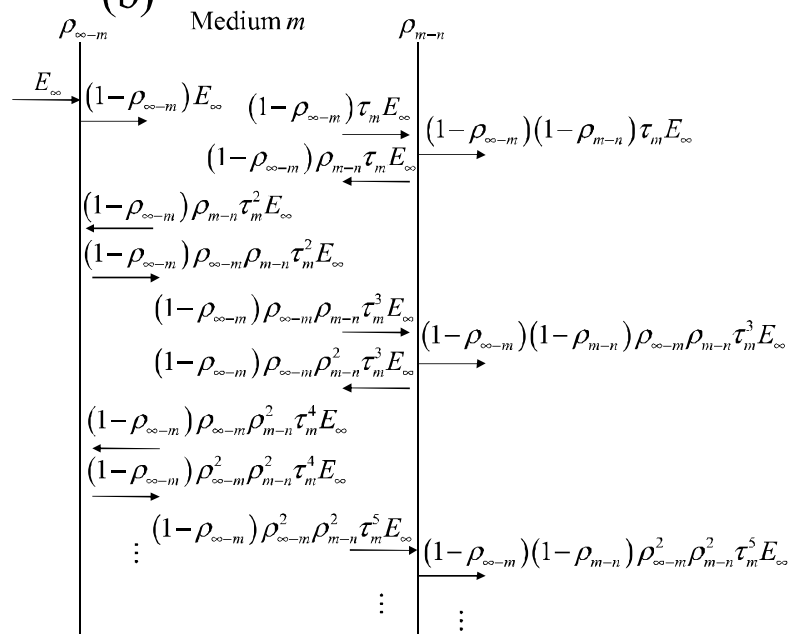

Fig. A3. Energy reflected and transmitted from medium $m$. (a) Apparent reflectivity. (b) Apparent transmissivity.

A.4. Energy emission by two-layer materials including reflection from ambient

Consider now the case shown in Fig. A4. The energy striking an IR camera is composed of four components: the energy emitted by an opaque surface $s$, emission from the two media ( $m$ and $n$ ), and by reflection from the surroundings.

For the special case where $\varepsilon_{s}=1$, we need to consider each of the four energy components below:

\section{A.4.1. Energy from surroundings}

The energy from the surroundings $\left(E_{\infty}=\sigma T_{\infty}^{4}\right)$ is reflected from surfaces $\infty-m$ and $m-n$ is given by $\rho_{\text {app, } \infty-m} E_{\infty}$. The energy transmitted through $m$ is partly attenuated in $n$ and the remainder is completely absorbed by surface $s$. No reflection occurs at surface $s$, simplifying the analysis.

\section{A.4.2. Energy emitted by $m$}

The energy emitted by $m$ leaving the interface $\infty-m$ is given by

$E_{m}=\int_{0}^{L_{m}} \alpha_{m} \sigma\left[T_{m}(x)\right]^{4} \exp \left(-\alpha_{m} x\right) d x$

The energy emitted by $m$ that leaves interface $m-n$ is again absorbed either by $n$ or $s$ and does not reach the camera.

\section{A.4.3. Energy emitted by $n$}

The energy emitted by $n$ that reaches interface $m-n$ is given by

$E_{n}=\int_{0}^{L_{n}} \alpha_{n} \sigma\left[T_{n}(x)\right]^{4} \exp \left(-\alpha_{n} x\right) d x$

The energy that is transmitted through $m$ and reaches the IR camera is given by $\tau_{a p p, \infty-m} E_{n}$.

\section{A.4.4. Energy emitted by s}

The energy emitted from $s\left(E_{s}=\sigma T_{s}^{4}\right)$ that reaches interface $m-n$ is $\tau_{n} E_{s}=\exp \left(-\alpha_{n} L_{n}\right) E_{s}$. The portion transmitted through $m$ that strikes the camera is $\tau_{a p p, \infty-m} \tau_{n} E_{s}$.

The total energy striking the camera is the sum of these four components and is given by

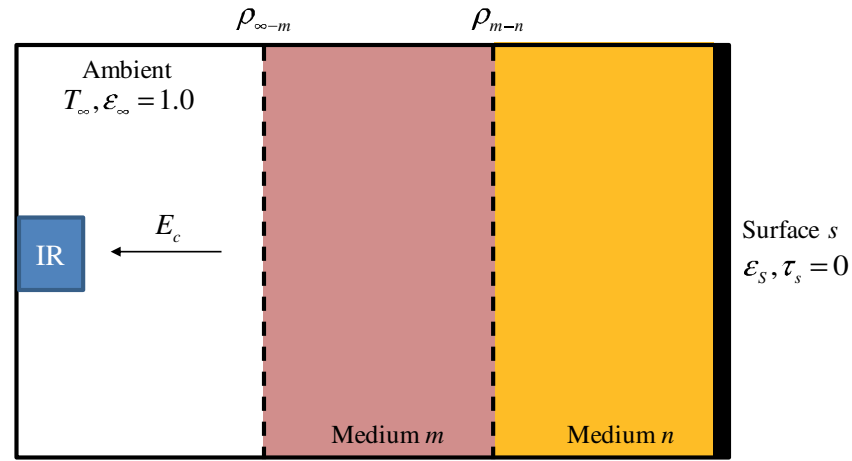

Fig. A4. Schematic diagram for two-layer materials.

$$
\begin{aligned}
E_{c}= & {\left[\rho_{\infty-m}+\frac{\left(1-\rho_{\infty-m}\right)^{2} \rho_{m-n} \tau_{m}^{2}}{1-\rho_{\infty-m} \rho_{m-n} \tau_{m}^{2}}\right] E_{\infty} } \\
& +\left[\frac{\left(1-\rho_{\infty-m}\right)\left(1+\rho_{m-n} \tau_{m}\right)}{1-\rho_{\infty-m} \rho_{m-n} \tau_{m}^{2}}\right] E_{m} \\
& +\left[\frac{\left(1-\rho_{\infty-m}\right)\left(1-\rho_{m-n}\right) \tau_{m}}{1-\rho_{\infty-m} \rho_{m-n} \tau_{m}^{2}}\right] E_{n} \\
& +\left[\frac{\left(1-\rho_{\infty-m}\right)\left(1-\rho_{m-n}\right) \tau_{m} \tau_{n}}{1-\rho_{\infty-m} \rho_{m-n} \tau_{m}^{2}}\right] E_{s}
\end{aligned}
$$

If the surface $s$ is not black $\left(\varepsilon_{s}<1\right)$ but still opaque, reflections from this surface must be accounted for and the problem become more complex since an infinite number of reflections occur within two materials. For the sake of brevity, only the final results are given below. The energy striking the camera accounting for infinite reflections from surface $s$ is given by

$E_{c}=\rho_{\infty-c} E_{\infty 1}+\varepsilon_{m-c} E_{m}+\varepsilon_{n-c} E_{n}+\tau_{s-c} E_{s}$

where

$$
\begin{aligned}
& \rho_{\infty-c}=\rho_{\infty-m}+\frac{\left(1-\rho_{\infty-m}\right)^{2} \rho_{a p p, m-n} \tau_{m}^{2}}{1-\rho_{\infty-m} \rho_{a p p, m-n} \tau_{m}^{2}} \\
& \varepsilon_{m-c}=\frac{\left(1-\rho_{\infty-m}\right)\left(1+\rho_{a p p, m-n} \tau_{m}\right)}{1-\rho_{\infty-m} \rho_{a p p, m-n} \tau_{m}^{2}}
\end{aligned}
$$




$$
\begin{gathered}
\varepsilon_{n-c}=\frac{\left(1-\rho_{\infty-m}\right)\left(1-\rho_{m-n}\right)\left(1+\rho_{n-s} \tau_{n}\right)}{\left(1-\rho_{\infty-m} \rho_{a p p, m-n} \tau_{m}^{2}\right)\left(1-\rho_{m-n} \rho_{n-s} \tau_{n}^{2}\right)} \\
\tau_{s-c}=\frac{\left(1-\rho_{\infty-m}\right)\left(1-\rho_{m-n}\right)\left(1-\rho_{n-s}\right) \tau_{m} \tau_{n}}{\left(1-\rho_{\infty-m} \rho_{a p p, m-n} \tau_{m}^{2}\right)\left(1-\rho_{m-n} \rho_{n-s} \tau_{n}^{2}\right)}
\end{gathered}
$$

and

$\rho_{a p p, m-n}-\rho_{m-n}+\frac{\left(1-\rho_{m-n}\right)^{2} \rho_{n-s} \tau_{n}^{2}}{1-\rho_{m-n} \rho_{n-s} \tau_{n}^{2}}$.

\section{Appendix B. Supplementary material}

Supplementary data associated with this article can be found, in the online version, at doi:10.1016/j.ijmultiphaseflow.2011.11.012.

\section{References}

Coney, M.W.E., 1973. The theory and application of conductance probes for the measurement of liquid film thickness in two-phase flow. J. Phys. E: Sci. Instrum. 6, 903. doi:10.1088/0022-3735/6/9/030.

Dupont, V., Thome, J.R., Jacobi, A.M., 2004. Heat transfer model for evaporation in microchannels. Part II: comparison with the database. Int. J. Heat Mass Transfer 47, 3387-3401.

Fischer, S., Herbert, S., Sielaff, A., Slomski, E.M., Stephan, P., Oechsner, M., 2011. Experimental Investigation of nucleate boiling on a thermal capacitive heater under variable gravity conditions. Microgravity Sci Technol. doi:10.1007/ s12217-011-9273-6.

Gerardi, C., Buongiorno, J., Hu, L., McKrell, T., 2010. Study of bubble growth in water pool boiling through synchronized, infrared thermometry and high-speed video. Int. J. Heat Mass Transfer 53 (19-20), 4185-4192.

Golobic, I., Petkovsek, J., Baselj, M., Papez, A., Kenning, D.B.R., 2009. Experimental determination of transient wall temperature distributions close to growing vapor bubbles. Heat Mass Transfer 45 (7), 857-866. doi:10.1007/s00231-0070295-.

Han, Y., Shikazono, N., 2009. Measurement of liquid film thickness in micro square channel. Int. J. Multiphase Flow 35 (10), 896-903. doi:10.1016/ j.ijmultiphaseflow.2009.06.006.
Kattan, N., Thome, J.R., Favrat, D., 1998a. Flow boiling in horizontal tubes. Part 1: Development of a diabatic two-phase flow pattern map. J. Heat Transfer 120, 140-147.

Kattan, N., Thome, J.R., Favrat, D., 1998b. Flow boiling in horizontal tubes. Part 2: New heat transfer data for five refrigerants. J. Heat Transfer 120, 148-155.

Kattan, N., Thome, J.R., Favrat, D., 1998c. Flow boiling in horizontal tubes. Part 3: Development of a new heat transfer model based on flow patterns. J. Heat Transfer 120, 156-165.

Klausner, J.F., Zeng, L.Z., Bernhard, D.M., 1990. Development of a film thickness probe using capacitance for asymmetrical two-phase flow with heat addition. Review of Scientific Instruments 53(5), 3147-3152, 1992, doi: 10.1063/ 1.1142568.

Krebs, D., Narayanan, V., Liburdy, J., Pence, D., 2010. Spatially resolved wall temperature measurements during flow boiling in microchannels. Exp. Thermal Fluid Sci. 34, 434-445. doi:10.1016/j.expthermflusci.2009.05.005.

Mani, P., Cardenas, R., Narayanan, V. 2012. Submerged Jet Impingement Boiling on a Nonuniformly Heated Polished Silicon Surface, Interpack2011-52042, ASME 2011 Pacific Rim Technical Conference and Exposition on Packaging and Integration of Electronic and Photonic Systems, Interpack 2011, Portland, OR, July 2011.

McMahon, H.O., 1950. Thermal radiation from partially transparent reflecting bodies. J. Opt. Soc. Am. 40 (6), 376-380.

Quinn, T.J., 1967. The calculation of the emissivity of cylindrical cavities giving near black-body radiation. Brit. J. Appl. Phys. 18, 1105-1113. doi:10.1088/0508$3443 / 18 / 8 / 310$.

Rouhani, Z., Axelsson, E., 1970. Calculation of void volume fraction in the subcooled and quality boiling regions. Int. J. Heat Mass Transfer 13, 383-393.

Shedd, T.A., Newell, T.A., 1998. Automated optical liquid film thickness measurement method. Rev. Sci. Instrum. 69 (12), 4205-4213.

Schweizer, N., Stephan, P., 2009. Experimental study of bubble behavior and local heat flux in pool boiling under variable gravity conditions. Multiphase Sci. Technol. 21 (4), 329-350. doi:10.1615/MultScienTechn.v21.i4.40.

Sefiane, K., Moffat, J.R., Mater, O.K., Craster, R.V., 2008. Self-excited hydrothermal waves in evaporating sessile drops. Appl. Phys. Lett. 93, 074103. doi:10.1063/ 1.2969072.

Shen, J., Graber, C., Liburdy, J., Pence, D., Narayanan, V., 2010. Simultaneous droplet impingement dynamics and heat transfer on nano-structured surfaces. Exp. Thermal Fluid Sci. 34, 496-503. doi:10.1016/j.expthermflusci.2009.02.003.

Thome, J.R., Dupont, V., Jacobi, A.M., 2004. Heat transfer model for evaporation in microchannels. Part I: presentation of the model. Int. J. Heat Mass Transfer 47, 3375-3385. 